\title{
Synchrony and Desynchrony in Integrate-and-Fire Oscillators
}

\author{
Shannon R. Campbell \\ Department of Physics, The Ohio State University, Columbus, Ohio 43210, U.S.A.
}

DeLiang L. Wang

Department of Computer and Information Science and Center for Cognitive Science, The Ohio State University, Columbus, Ohio 43210, U.S.A.

\section{Ciriyam Jayaprakash}

Department of Physics, The Ohio State University, Columbus, Ohio 43210, U.S.A.

Due to many experimental reports of synchronous neural activity in the brain, there is much interest in understanding synchronization in networks of neural oscillators and its potential for computing perceptual organization. Contrary to Hopfield and Herz (1995), we find that networks of locally coupled integrate-and-fire oscillators can quickly synchronize. Furthermore, we examine the time needed to synchronize such networks. We observe that these networks synchronize at times proportional to the logarithm of their size, and we give the parameters used to control the rate of synchronization. Inspired by locally excitatory globally inhibitory oscillator network (LEGION) dynamics with relaxation oscillators (Terman \& Wang, 1995), we find that global inhibition can play a similar role of desynchronization in a network of integrate-and-fire oscillators. We illustrate that a LEGION architecture with integrate-and-fire oscillators can be similarly used to address image analysis.

\section{Introduction}

Different features of visual objects appear to be processed in different cortical areas (Zeki, 1993). How these features are linked to form perceptually coherent objects is known as the feature binding problem. Theoreticians have proposed that correlations in the firing times of neurons may encode the binding between these neurons (Milner, 1974; von der Malsburg, 1981). A considerable amount of neurophysiological evidence supports this conjecture of temporal correlation (for a review see Singer \& Gray, 1995; also see Livingstone, 1996).

Based on the experimental findings, many oscillator networks have been proposed in which synchronous oscillations link features together (synchrony implies the same frequency and phase). This particular form of temporal correlation was called oscillatory correlation (Wang \& Terman, 1995). In 
oscillatory correlation two issues need to be addressed. The first is the need to achieve synchrony quickly in locally coupled networks. Our usage of the word quickly refers to a time of a few periods. This is based on biological data, which indicate that synchronous firings of neural groups begin two to three periods after the onset of stimulus (Singer \& Gray, 1995). Locally coupled networks are emphasized because a network with all-to-all coupling does not maintain pertinent geometrical and spatial information that is critical for perceptual processing (for further explanations see Sporns, Tononi, \& Edelman, 1991; Wang, 1993). The second issue is how to desynchronize the phases of different objects rapidly and robustly so that segmentation occurs.

In this article we study integrate-and-fire oscillators, possibly the simplest model of neuronal dynamics. A single variable represents the membrane potential. When this variable attains a certain threshold, it is said to fire, and it is reset to zero. When the oscillator fires, it sends excitation to its neighbors. Integrate-and-fire oscillators have frequently been studied as models of neuronal behavior (Peskin, 1975; Mirollo \& Strogatz, 1990). Several authors have noted the ability of locally coupled networks to synchronize (Mirollo \& Strogatz, 1990; Corral, Perez, Diaz-Guilera, \& Arenas, 1995; Hopfield \& Herz, 1995). However, it is not known how quickly these networks synchronize. In computational terms, the time complexity of synchronization in these networks is unknown.

In one of the few studies systematically addressing locally coupled integrate-and-fire oscillators, Hopfield and Herz (1995) reported that a twodimensional locally connected integrate-and-fire oscillator network (40 $\times$ 40 ) with excitatory couplings exhibits global synchrony (all oscillators fire in unison) on long timescales (about 100 periods). Due to its convergence speed, global synchrony was considered to be too slow to underlie biological information processing. When examining this phenomenon, we found that synchrony in the same size network can actually be achieved quickly (in two to three periods) through appropriate adjustment of parameters. Further numerical investigations revealed a surprising scaling relation: the average time to synchrony increases as the logarithm of the system size in both one-dimensional (1D) and two-dimensional (2D) systems.

Given that locally coupled integrate-and-fire oscillators synchronize quickly, we have already attained one of the aspects of oscillatory correlation: fast synchronization. The other aspect of oscillatory correlation is desynchronization. In order to desynchronize different groups of oscillators while maintaining synchrony within each group, we use the locally excitatory globally inhibitory oscillator network (LEGION) architecture proposed by Terman and Wang (1995). This architecture relies on a single inhibitory unit, which is coupled to every oscillator, to desynchronize different groups of oscillators. To illustrate the potential of this network, we provide results on some image segmentation tasks.

We define a system of integrate-and-fire oscillators in section 2.1. We then describe the behavior of two interacting integrate-and-fire oscillators in sec- 
tion 2.2. We display our data indicating that the time to synchrony scales as the logarithm of the system size for 1D and 2D systems in section 3; we also describe how the system parameters are related to the rate of synchronization in this section. In section 4 we describe how we create a LEGION network with integrate-and-fire oscillators that can desynchronize multiple groups of oscillators while maintaining synchrony within each group using binary images. In section 5 we modify and extend this network so that gray-level images can be processed. We demonstrate the potential of this network by segmenting real images. Section 6 provides further discussions.

\section{Model Description and Behavior}

2.1 Model Definition. A network of integrate-and-fire oscillators is defined as

$$
\dot{x}_{i}=-x_{i}+I_{0}+\sum_{j \in N(i)} J_{i j} P_{j}(t), \quad i=1, \ldots, n,
$$

where the sum is over the oscillators in a neighborhood, $N(i)$, about oscillator $i . x_{i}$ represents some voltage-like variable that we call the potential of oscillator $i$. The parameter $I_{0}$ controls the period of an uncoupled oscillator. The threshold of an oscillator is set to 1 . When $x_{i}=1$ the oscillator is said to fire; its potential is instantly reset to 0 , and it sends excitation to its neighbors.

The interaction between oscillators, $P_{j}(t)$, is defined as

$$
P_{j}(t)=\sum_{m} \delta\left(t-t_{j}^{m}\right)
$$

where $t_{j}^{m}$ represents the $m$ firing times of oscillator $j$ and $\delta(t)$ is the Dirac delta function. When oscillator $j$ fires at time $t$, oscillator $i$ receives an instantaneous pulse. This pulse increases $x_{i}$ by $J_{i j}$. If $x_{i}$ is increased above the threshold, it will fire. Note that information is transmitted between oscillators instantaneously, and thus the propagation speed is infinite.

The coupling is between nearest neighbors; that is, an oscillator interacts with two neighbors in $1 \mathrm{D}$ and four neighbors in $2 \mathrm{D}$. The connection strength from oscillator $j$ to oscillator $i$ is normalized as

$$
J_{i j}=\frac{\alpha}{Z_{i}}
$$

where $Z_{i}$ is the number of nearest neighbors that oscillator $i$ has, for example, $Z_{i}=2$ for an oscillator $i$ at the corner of a 2D system. The constant $\alpha$ is the coupling strength. The normalization ensures that all oscillators receive the same amount of stimulus and therefore have the same trajectory in 
phase space when synchronous (Wang, 1995). As Wang $(1993,1995)$ pointed out, such weight normalization is critical for synchronization in less homogeneous situations, such as open boundary conditions; it has been used in later studies (Hopfield \& Herz, 1995; Traub, Whittington, Stanford, \& Jefferys, 1996). Note that there are only two parameters in system 2.1: the coupling strength $\alpha$ and $I_{0}$. When oscillator $i$ reaches its threshold, it will fire, and its value will be reset to zero. Oscillator $i$ then sends an instantaneous impulse to neighboring oscillator $j$. If oscillator $j$ is induced to fire, then its value is reset in the following manner:

$$
x_{j}\left(t^{+}\right)=x_{j}\left(t^{-}\right)+J_{j i}-1
$$

Since oscillator $j$ fires, oscillator $i$ immediately receives excitation and thus $x_{i}\left(t^{+}\right)=J_{i j}$. Because of this, the period of the synchronous system is shorter than the period of a single uncoupled oscillator. The synchronous period of the system is given by

$$
\log \left(\frac{I_{0}-\alpha}{I_{0}-1}\right) .
$$

Hopfield and Herz (1995) called this particular realization of a network of integrate-and-fire oscillators Model A.

2.2 A Pair of Integrate-and-Fire Oscillators. We now describe the behavior of a pair of integrate-and-fire oscillators. This section contains a short summary of some of the results that Mirollo and Strogatz (1990) derived. The trajectory of a single uncoupled oscillator can be solved analytically$x(\phi)=f(\phi)=I_{0}(1-\exp (-\gamma \phi))$, where $\gamma=\log \left(I_{0} /\left(I_{0}-1\right)\right)$, the period of the oscillator, and $\phi$ can be thought of as a phase, or a local time variable. Note that the function $f(\phi)$ increases monotonically $\left(f^{\prime}(\phi)>0\right)$ and is concave down $\left(f^{\prime \prime}(\phi)<0\right)$. Mirollo and Strogatz (1990) showed that an all-to-all connected system of integrate-and-fire oscillators with positive pulsatile coupling as well as $f^{\prime}(\phi)>0$ and $f^{\prime \prime}(\phi)<0$, synchronizes. We display the temporal evolution of a pair of integrate-and-fire oscillators in Figure 1. The oscillators initially have different potentials, but the interaction quickly adjusts their trajectories so that they eventually fire in unison. When two or more oscillators fire at the same time, we call them synchronous. The spikes shown in Figure 1 when an oscillator reaches the threshold are for illustrative purposes only.

Using $f(\phi)$ and its inverse, $g(x)$, one can calculate the return map (see Figure 2A) for a pair of pulse coupled integrate-and-fire oscillators. A line of slope 1 is also shown in Figure 2A for comparison. The horizontal axis represents the initial phase difference between the two oscillators, and the vertical axis represents the phase difference between the two oscillators after they have both fired once. There are three different regions in the return 


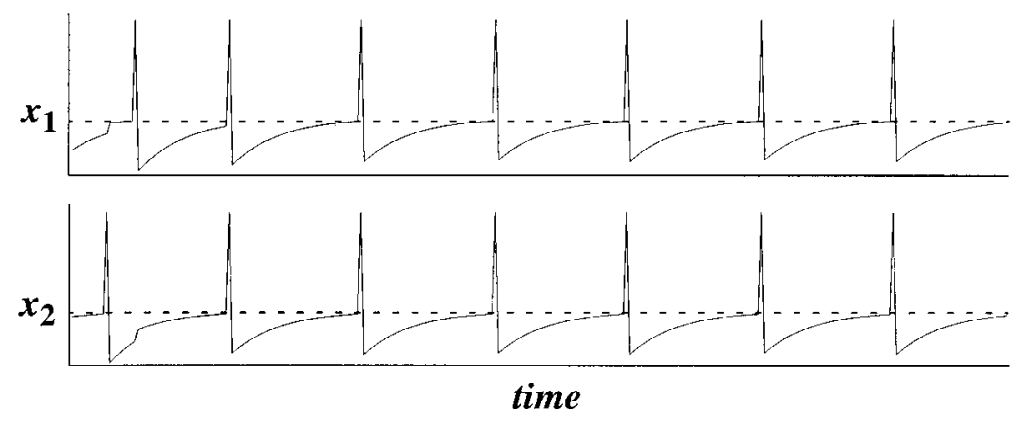

Figure 1: Diagram of a pair of integrate-and-fire oscillators with pulsatile coupling. The solid curves represent the potentials of the two coupled oscillators and the dashed lines represent the threshold. The initial potentials of the oscillators are chosen randomly. The oscillator labeled $x_{2}$ fires first, and the potential of $x_{1}$ increases at that time. Similarly, when $x_{1}$ fires, the potential of $x_{2}$ increases. The phase shifts caused by the pulsatile interaction cause the oscillators to fire synchronously by the second cycle.
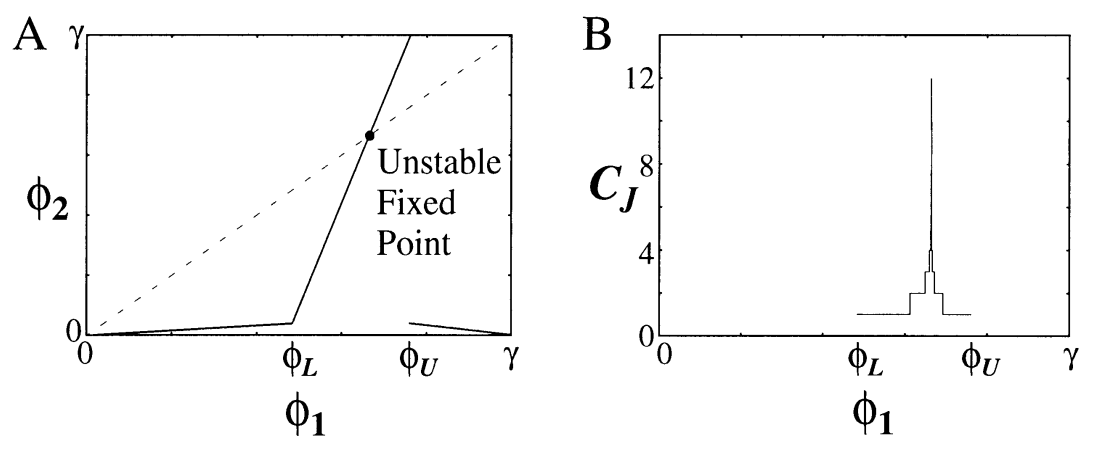

Figure 2: (A) Return map for two pulse-coupled integrate-and-fire oscillators. The phase difference between the oscillators before they have jumped $\left(\phi_{1}\right.$, horizontal axis) and after they have jumped ( $\phi_{2}$, vertical axis). (B) Plot of the number of cycles needed, $C_{J}$, before the two oscillators are synchronous as a function of $\phi_{1}$. Both plots use $I_{0}=1.11$ and $\alpha=0.2$.

map. The first region is in the range of initial conditions $\phi_{1} \in\left[0, \phi_{L}\right]$, where $\phi_{L}=1-g(1-\alpha)$. In this region, the oscillators are near enough so that when one oscillator fires, the second oscillator is induced to fire as well. We call this the jumping region, and it has a direct analog in a pair of relaxation oscillators (Somers \& Kopell, 1993). Once the two oscillators are in the 
jumping region, they always fire at the same time, and it can be shown that their phase difference always decreases. The second region is in the range of initial conditions from $\left[\phi_{L}, \phi_{U}\right]$, where $\phi_{U}=1-g\left(f\left(\phi_{L}\right)-\alpha\right)$. For these initial conditions, when the first oscillator fires, the other oscillator receives excitation, but is not induced to fire at the same time (as in the first firing of $x_{2}$ in Figure 1). Similarly, when the second oscillator fires, the relative phase between the two oscillators again changes, but the two oscillators do not fire in unison. In this region there is an unstable fixed point for which the phase between the oscillators does not change. In the third region, the first oscillator fires and the second oscillator receives excitation but does not fire immediately. When the second oscillator fires, the first oscillator receives excitation and is induced to fire a second time. We consider this third region part of the jumping region. In summary, this return map contains a range of initial conditions for which the two oscillators fire together and another set of initial conditions for which it may take several cycles before both oscillators begin firing together.

In Figure 2B we display the number of cycles needed before the two oscillators are in the jumping region. The horizontal axis in Figure 2B indicates the initial phase separation between the two integrate-and-fire oscillators, and the vertical axis indicates the number of cycles needed until the two oscillators are in the jumping region. As expected, initial conditions near the unstable fixed point require more cycles before synchrony occurs.

The derivative of the return map at the unstable fixed point is given by

$$
\left(1+\frac{\alpha}{I_{0}}\left\{\frac{\alpha+\sqrt{\alpha^{2}+4 I_{0}\left(I_{0}-1\right)}}{2\left(I_{0}-1\right)}\right\}\right)^{2} .
$$

This quantity gives one indication how repulsive the unstable fixed point is; furthermore, the linearity of the second region in the return map of Figure $2 \mathrm{~A}$ is obtained for a wide range of $I_{0}$, and hence the derivative at the fixed point. Therefore the derivative may indicate how fast the system approaches the stable synchronous solution. The fixed point is unstable for all positive values of $\alpha$ and all values of $I_{0}>1$. For $I_{0} \gg 1$ the fixed point is still unstable but the derivative is near 1 , indicating a relatively slow approach to synchrony. When $I_{0}$ decreases the derivative increases, indicating a faster approach to synchrony. We will compare equation 2.6 to the rate of synchronization in networks of oscillators in section 3.

The rate of synchronization, particularly phase compression between oscillators that have fired simultaneously, depends on the concavity of the oscillator trajectory in a more direct way. With $f(\phi)$ concave down, the oscillators approach the threshold at a decreasing speed, and thus the time difference between the two oscillators near the threshold can be quite large while the difference in their potentials is quite small. On the other hand, the time difference near the reset can be quite small while the potential 
difference is quite large. When the two oscillators fire synchronously, their potential difference is kept constant right before and after the firing while their time difference decreases. This results in a phase compression between the oscillators, the amount of which is determined by the concavity. This analysis is similar to an earlier analysis by Somers and Kopell (1993) on a pair of relaxation oscillators, where the concavity of nullclines plays an analogous role (see Terman \& Wang, 1995, for a similar analysis for a network of relaxation oscillators).

\section{Synchrony in Integrate-and-Fire Oscillator Networks}

We have observed that the average time to synchrony increases as the logarithm of the system size in both 1D and 2D noiseless systems for random initial conditions. Our observations are based on many trials of oscillator networks that were numerically integrated with an event-driven algorithm. For all data shown, we used the following procedure:

1. The potentials are chosen from the range $[0,1]$.

2. Find the oscillator nearest to the threshold. The amount of time it needs to fire is calculated and all the oscillators are advanced using this amount of time.

3. The oscillator at the threshold fires. The potential of this oscillator is reset to zero, and the potentials of its neighboring oscillators are increased using equation 2.3.

4. Check if any of the oscillators that have received excitation are above the threshold. If any oscillators are above the threshold, they are reset according to equation 2.4, and excitation is sent to their neighbors. Repeat this step until no oscillators are above the threshold.

5. Return to step 2.

All trials with locally coupled networks of integrate-and-fire oscillators have resulted in synchrony. Over $10^{5}$ trials in which the initial conditions were chosen randomly and uniformly in the range $[0,1]$ have been recorded. These networks were also tested with other, more correlated initial conditions. Networks in which the initial conditions were spin waves also achieved synchrony. The speed with which networks with spin wave-type initial conditions attained synchrony was, on average, faster than that using random initial conditions. For long-wavelength spin waves, the potentials of the oscillators are near to each other, and one oscillator can cause many of its neighbors to fire. Several large groups, or blocks, of oscillators form and fire synchronously during the first cycle. For short wavelengths that are integer multiples of the lattice size, the oscillators also synchronize more quickly than with random initial conditions. Small blocks of synchronous oscillators form, and since these blocks are formed based on repeating 
patterns of initial conditions, they also have a spatially repeating pattern. This process repeats until synchrony occurs. This implies that incommensurate wavelengths may take longer to synchronize because spatially repeating patterns of blocks do not form and their interactions with one another would not be uniform. This intuition does appear to be correct; incommensurate wavelengths tend to have longer synchronization times. However, we could not find any initial conditions whose resultant time to synchrony was an order of magnitude larger than the average time to synchrony with random initial conditions (over $10^{4}$ incommensurate frequencies were tested). Similar tests in 2D networks yield similar results. There are a few solutions that are not synchronous - for example, initial conditions in which the phase difference between neighboring pairs of oscillators is at the unstable fixed point shown in Figure 2A. In numerical tests with these initial conditions, floating-point errors eventually cause small perturbations away from this unstable solution, and synchrony quickly results. Furthermore, in trials with periodic boundary conditions (a ring topology), solutions with traveling waves were never observed. Based on these extensive observations, we conclude that locally coupled networks of integrate-and-fire oscillators always synchronize. Although all of our data have been gathered using one or two specific integrate-and-fire oscillators, we claim that our results generalize to the class of integrate-and-fire oscillators with positive coupling, $f^{\prime}(\phi)>0$, and $f^{\prime \prime}(\phi)<0$.

3.1 One-Dimensional Systems. We display the temporal evolution of a 1-D network in Figure 3. The figure shows the firing times of all the oscillators in a network of 400 oscillators. Time is shown along the vertical axis, and the horizontal axis represents the index of the oscillators. Each dot represents the firing time of one oscillator, and each line represents the firing time of a block of oscillators. Near the bottom of the graph, there are many single dots and small lines. These represent the fact that the oscillators have random initial conditions and initially have distinct firing times. But quickly, by the time $t=5$, blocks of various sizes have formed. Just after time $t=5$, at the lower left of Figure 3, oscillators 1-20 fire simultaneously. This block formed from three smaller blocks. Near $t=30$ there is a single solid line shown, indicating that all the oscillators fired at the same time. Underneath this line are two separate blocks of oscillators. One might at first wonder why these two large blocks have merged in just one cycle. This represents the fact that the system has an instantaneous propagation speed. When the oscillator at the left border of the right block receives excitation, it is induced to fire. When this oscillator fires, it sends excitation to its right neighbor, which is also induced to fire, and this process repeats throughout the length of the right block. In the algorithm we use, the firing and reset of an oscillator are instantaneous, as are the excitatory pulses sent to neighboring oscillators. This results in an infinite propagation speed. Thus, no matter how large a block is, it can merge with a neighboring block in one 


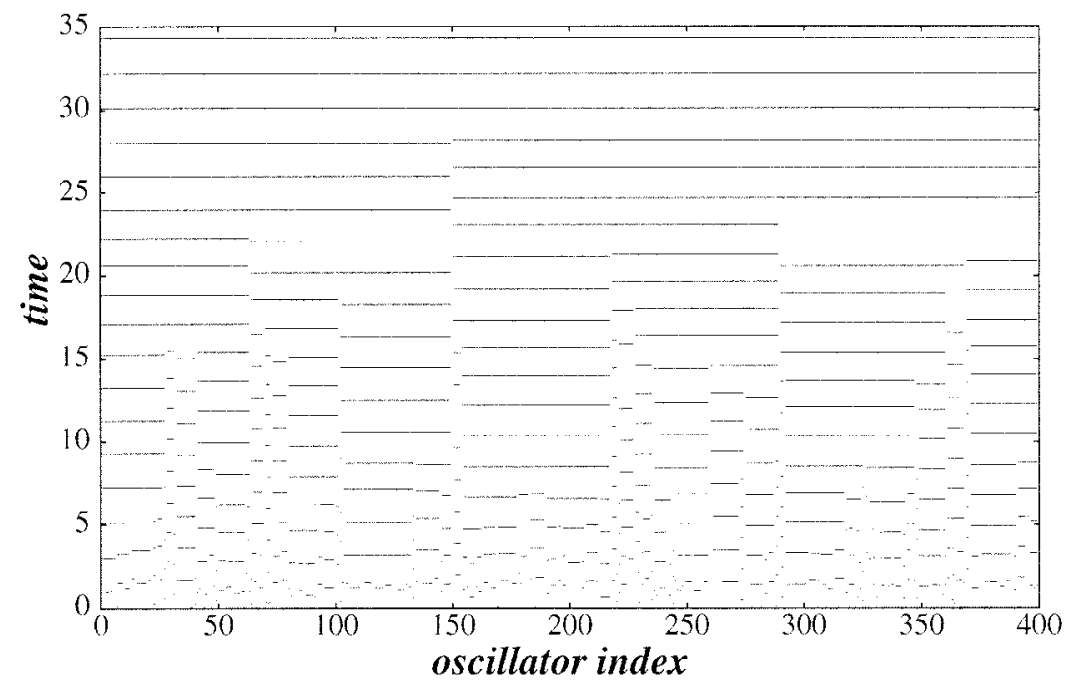

Figure 3: Diagram displaying the evolution of a 1D network of 400 integrateand-fire oscillators. The vertical axis represents time, and the horizontal axis represents the position of the oscillator in the chain. Each dot represents the firing time of a single oscillator, and each line represents that of a block of oscillators. The parameters are $\alpha=0.2, I_{0}=1.11$.

cycle. The most striking feature of Figure 3 is that it is impossible to find an increase in the number of blocks. In fact, as shown in the appendix, the number of synchronized blocks never increases.

In Figure 4 we display data indicating that the time needed to synchronize a chain of size $n$ oscillators increases in proportion to $\log _{10}(n)$. Time is shown in units of periods. The averages are based on several hundred trials with random initial conditions. The averages appear to lie on a straight line for each of the three parameter pairs tested. Although only three data sets are displayed, our tests with other parameters yield a change only in the slope of the resulting line. The inset in this figure is shown to indicate the standard deviation of the averages. The standard deviations for the other data sets are similar in that they remain nearly constant after the chain length becomes larger than 20. We tested various combinations of $\alpha$ and $I_{0}$ in the ranges $\alpha \in[0.0025,0.96]$ and $I_{0} \in[1.01,20]$; all tested parameters resulted in a logarithmic relationship. In section 3.3 we discuss how these two parameters relate to the slopes of the lines shown in Figure 4 . We note that only several hundred trials were needed to compute the averages because our simulations indicated that the distribution of the synchronization times did not have a long tail (Campbell, 1997). 


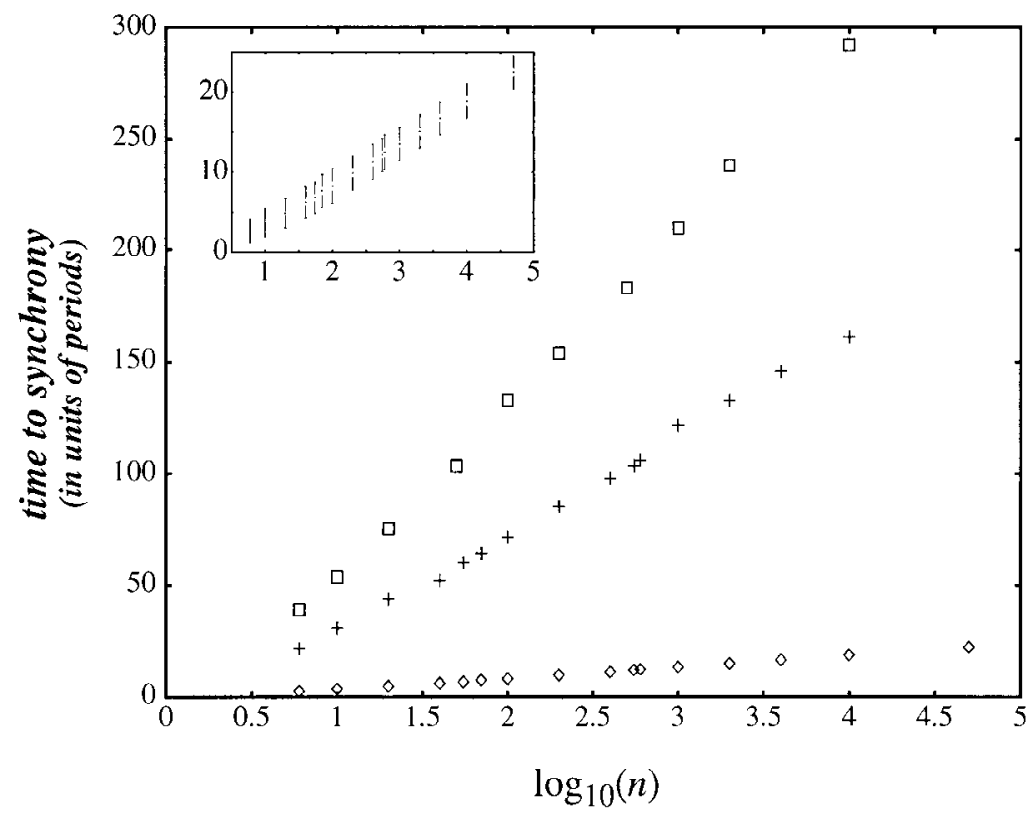

Figure 4: Average time needed for a chain of $n$ oscillators to synchronize as a function of $\log _{10}(n)$. Three symbols represent different parameters: squares: $\alpha=0.48, I_{0}=10$; plus signs: $\alpha=0.025, I_{0}=1.1$; diamonds: $\alpha=0.2, I_{0}=1.11$. The data are based on approximately 300 trials with random initial conditions. The inset displays the diamond data along with the standard deviation of the averages.

A heuristic understanding for our numerical results is as follows. As is done typically in 1D problems in statistical mechanics, we focus on the domain walls between adjacent clusters (blocks) of sites that are synchronized. As shown in the appendix, the number of domain walls, or equivalently the number of oscillator blocks, does not increase, so the only dynamically relevant process for a domain wall between two clusters is its disappearance when the two clusters become synchronized; the cluster that fires first sends a pulse to its neighboring clusters, which then may also fire depending on the difference in the dynamical variables of the two neighboring clusters. Since each domain wall has a nonzero probability of disappearing per unit time, one would expect that the walls disappear at a constant rate when averaged over the ensemble of initial conditions. Such a nonvanishing mean rate, $r$, for the removal of the domain walls automatically implies that the number of domain walls decreases as $\exp (-r t)$ and the entire system becomes synchronized in a time proportional to the logarithm of the initial 


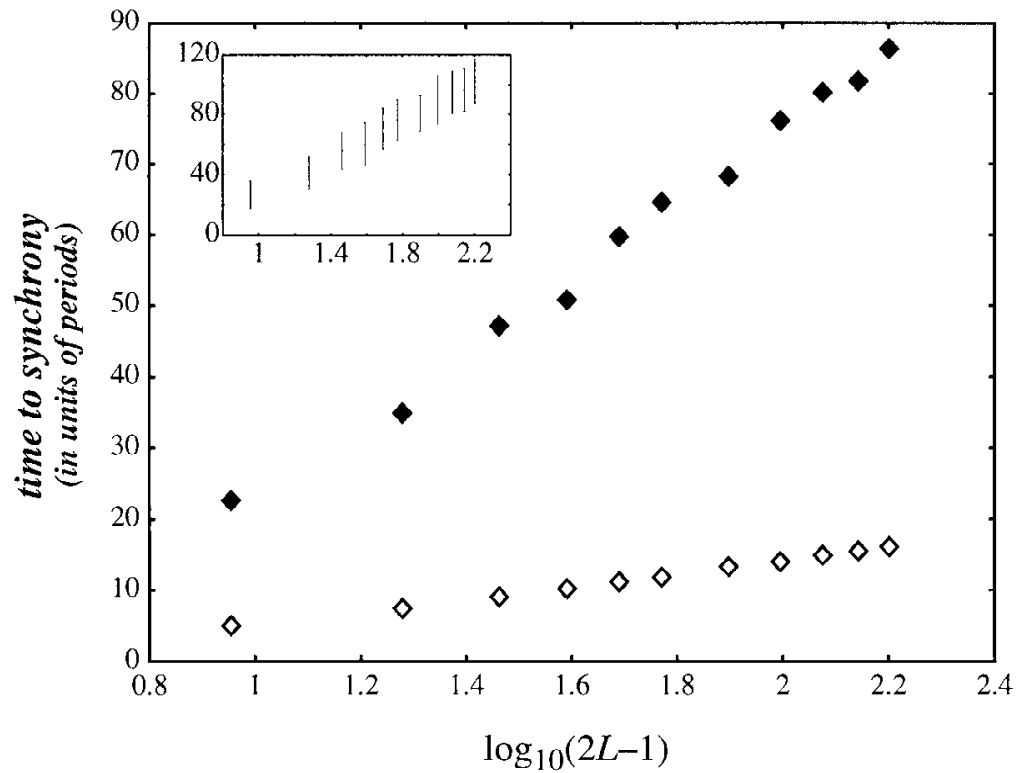

Figure 5: Average times for an $L \times L$ network of oscillators to synchronize are plotted as a function of $\log _{10}(2 L-1)$. The solid diamonds are for the parameters $\alpha=0.2, I_{0}=2.0$, and the open diamonds are for $\alpha=0.2, I_{0}=1.11$. Each average is computed from approximately 100 trials with random initial conditions. The inset indicates the standard deviation for the solid diamond data.

number of domain walls. For the initial conditions we have considered, the number of domain walls is proportional to the size of the chain, and this gives a heuristic explanation of our numerical results.

3.2 Two-Dimensional Systems of Oscillators. We display the average synchronization time for a 2D system as a function of $\log _{10}(2 L-1)$ in Figure 5 , where the system size is $L \times L$. Time is again shown in units of periods. In this $2 \mathrm{D}$ system, each oscillator is coupled to its four nearest neighbors, and the longest distance between any two oscillators (in terms of lattice sites) is $2 L-1$. The data indicate that the average time to synchrony scales logarithmically with the system size. We have tested more parameters than shown in Figure 5, and all tested parameters yield an identical scaling relation. The inset indicates the standard deviation for one set of data. Again, other sets of data show similar patterns of the standard deviation.

All trials with 2D networks resulted in synchrony. We tested various size spin waves in the two directions and obtained similar results to those in 
1D systems: synchrony was achieved regardless of the initial conditions. Traveling waves, rotating waves, or other desynchronous solutions were never observed, even with periodic boundary conditions.

Unlike in 1D systems, we do not analytically know whether the number of synchronized oscillator blocks does not increase in 2D. The situation in 2D is considerably more complex; for example, it is possible that an oscillator is not recruited to fire the first time it receives a pulse from one of its neighbors but can after more of its neighbors have jumped. In our simulations, we have not found a case where the number of blocks increases, which suggests that our heuristic interpretation for 1D may carry over to 2D. We note that even if the number of blocks increases occasionally, logarithmic scaling may still hold because what matters for synchronization speeds is how grouping of oscillator blocks dominates breaking if the latter does occur. Note also that for 2D systems, blocks have more interaction paths and thus are more conducive to synchronization.

We found that synchrony in the same size network as simulated in Hopfield and Herz (1995) can be achieved rapidly (in two to three periods) by using different parameter values. We also tested integrate-and-fire oscillator networks with the same parameter values used in Hopfield and Herz (1995). We confirmed their simulation results that a $40 \times 40$ network with $I_{0}=10$ and $\alpha=0.96$ resulted in an average time to synchrony of approximately 100 periods. We also tested these parameters with different size networks and found that, although it was very slow for a $40 \times 40$ network, the average time to synchrony still held a logarithmic relation with the system size. In addition, we tested equivalent parameters in 1D systems and again found the logarithmic scaling relation (see the squares in Figure 4). Thus, the reason that Hopfield and Herz (1995) did not observe rapid global synchrony is that the specific parameter values they used are not good for fast synchrony.

As we examine 2D systems, a natural question is how the rate of synchronization varies as the dimension of the system changes. We first define the rate of synchronization as follows. The data indicate that $\left\langle T_{S}\right\rangle \sim \frac{1}{r_{S}} \log (n)$, where $\frac{1}{r_{S}}$ corresponds to the slope of a line from Figures 4 and 5 . We refer to $r_{S}$ as the rate of synchronization. In tests where the value of $\alpha$ is held constant but the dimension of the system changes from 1 to 2 , we find that the rate of synchrony halves. When the individual coupling strengths between oscillators are maintained $-\alpha$ doubles as the system dimension increases from 1 to 2-we find that the rate of synchronization remains approximately the same between $1 \mathrm{D}$ and 2D systems. This indicates that the rate of synchronization is controlled by the individual connection weights between oscillators and not the total connection weights to each oscillator.

3.3 Rate of Synchronization. We now describe how the rate of synchronization is related to the system parameters. It is reasonable to expect that 
the overall scale is set by the behavior of a pair of oscillators; the derivative of the return map given by equation 2.6 describes the rate at which the two oscillators are repelled from the unstable fixed point after one iteration. In continuous time (measured in units of the period of an oscillator), the rate can be approximated by an exponential $\left(1+r_{S}\right)^{2} \approx \exp \left(2 r_{S}\right)$ and thus $r_{S}$, defined by

$$
r_{S}=\frac{\alpha_{S}}{I_{0}}\left\{\frac{\alpha_{S}+\sqrt{\alpha_{S}^{2}+4 I_{0}\left(I_{0}-1\right)}}{2\left(I_{0}-1\right)}\right\},
$$

can be used to set the rate scale to measure synchrony. In equation $3.1, \alpha_{S}$ represents the single connection strength between a pair of oscillators in the network, as opposed to the total connection strength, which is given by $\alpha$. In Figure 6 we show a scatter plot of actual rates of synchronization computed from numerical simulations with respect to $r_{S}$ given by equation 3.1. The figure shows good proportionality between the equation and the measured rates of synchrony (the majority of points lie along a straight line). Note that the rates of synchrony for this figure range from 0 to 1 , which implies that we tested a wide range of parameters. Rates of synchrony near 0 yield extremely slow synchronization rates, and a rate of synchrony near 1 means that 10 cycles are enough to synchronize a chain of $10^{10}$ oscillators.

Several data points in Figure 6 exhibit a significant deviation from the straight line. The majority of these points that are not along the line result from values of the coupling strength that are greater than 0.8 . This is as expected because as $\alpha$ nears 1, the period of the oscillator system approaches 0 ; the oscillators fire frequently but change their relative phase only slowly. As $\alpha$ nears 1 , the time to synchrony becomes infinite. At $\alpha=1$ system equation 2.1 is meaningless because the oscillators are constantly firing and resetting. Our data reflect this understanding because as the coupling becomes greater than 0.8 , approximation 3.1 becomes worse. Our data also indicate that this approximation is not good for values of $I_{0}<1.05$. Note that our argument for using $r_{S}$ to set the rate scale is valid if the fixed point is in the middle of the second region (see Figure 2A). As $I_{0}$ becomes smaller than 1.05, the derivative gets very large, and the second region becomes very narrow, and so the phase difference gets into the jumping region (the first region or the third region) rapidly. Since the phase difference does not spend enough time in the vicinity of the fixed point, we think that the rate of deviation from the fixed point no longer sets the scale reliably. In this case, rapid synchronization is mainly accounted for by the behavior in the jumping region (the first and the third region).

3.4 Heterogeneity. We have studied the behavior of the system with heterogeneity in the intrinsic frequencies. When the variations are bounded- 


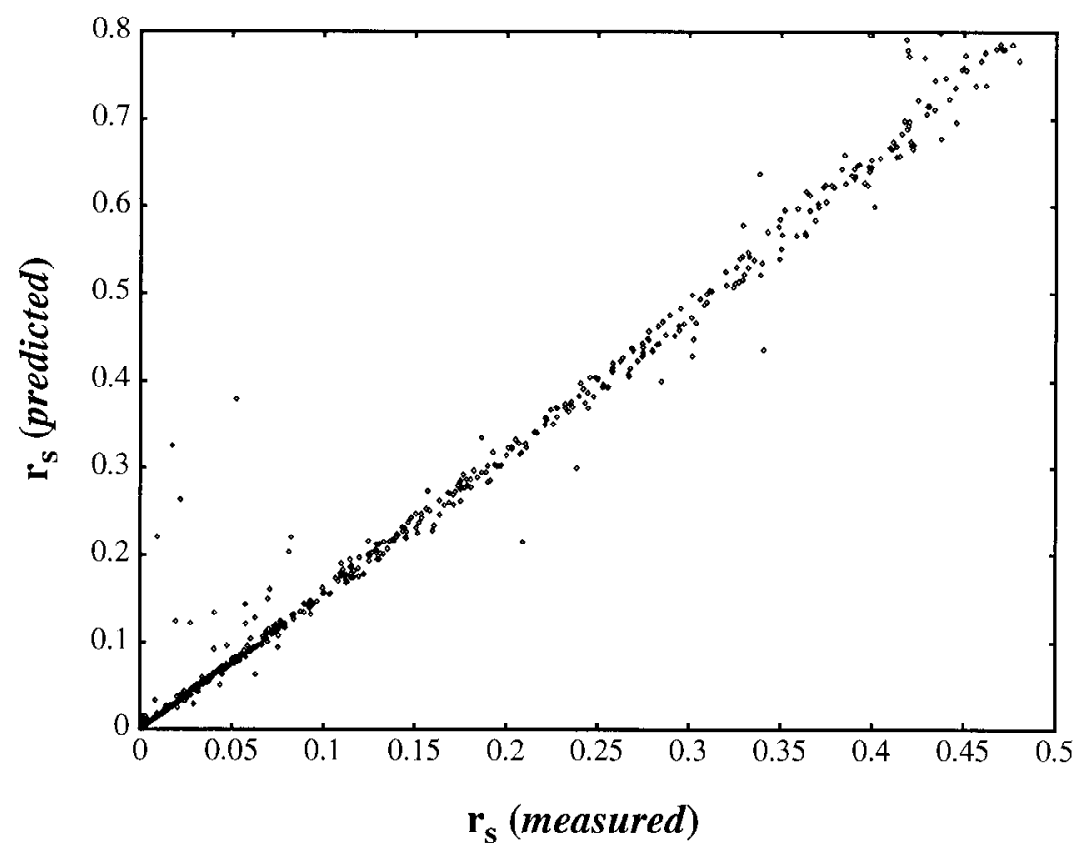

Figure 6: Scatter plot of the predicted rate of synchrony from equation 3.1 against the measured value. The measured rates of synchrony were obtained from oscillator chains by randomly choosing $n$ (from 150 to 1000), the coupling strength (from 0 to 1 ), and $I_{0}$ (from 1 to 100), and calculating the average time to synchrony using 100 trials with random initial conditions. The figure shows the results with approximately 575 different parameter choices.

for example, within 5\%-synchrony is still achieved in both $1 \mathrm{D}$ and $2 \mathrm{D}$ systems. When the distribution of frequencies is gaussian, the network can achieve synchrony only if the variance is small and the system size is modest (say, 100 in a 1D chain). The oscillators do not follow the same path in phase space since their speeds depend on the frequency; nevertheless, with a sufficiently small difference in frequencies, when the fastest oscillator jumps, it can induce the rest of the network to fire simultaneously. For long chains or larger variances of intrinsic frequencies, the chain evolves to clusters of synchronous oscillators. The border between clusters contains neighboring oscillators whose intrinsic frequence difference is too large for them to fire together; this occurs due to the tails in the gaussian distribution. We have not studied the effects of heterogeneity systematically. In particular, the effect on the rate of synchronization has not been investigated. 


\section{Desynchrony}

The locally coupled networks of integrate-and-fire oscillators have been shown to have the property that synchrony is quickly achieved. But a system that only achieves synchrony is not very useful for information processing, since such a system is dissipative and almost all information is lost. In order to perform computations, some other mechanisms must exist that can store or represent information. In oscillatory correlation, the different phases of oscillators encode binding and segregation information.

In order to create a network of integrate-and-fire oscillators for oscillatory correlation, we need a mechanism that desynchronizes different oscillator groups. Such a mechanism would need to be long range since the phases of different oscillator groups need to be desynchronous regardless of their positions in the network. Our construction employs a global inhibitor, and the architecture of our network is identical to the LEGION networks proposed by Terman and Wang (1995). The main difference is that the basic unit in our network is an integrate-and-fire oscillator rather than a relaxation oscillator. Figure 7A displays a diagram of the LEGION architecture.

We now define a LEGION network that uses integrate-and-fire oscillators as its basic units. The activity of each oscillator in the network is described by

$$
\dot{x}_{i}=-x_{i}+I_{i}+\sum_{j \in N(i)} J_{i j} P_{j}(t)-G(t),
$$

where $N(i)$ represents the four nearest neighbors of oscillator $i$. The parameter, $I_{i}$, is now dependent on the input image; we refer to this parameter as the stimulus given to oscillator $i$. In this section we discuss binary images. The respective stimulus for each oscillator is either $I_{i}>1$ or $I_{i}=0$. If $I_{i}>1$ we call oscillator $i$ stimulated. If an oscillator does not receive stimulus, $I_{i}=0$, its potential decays exponentially toward zero. As before, the threshold for each oscillator is 1 . The interaction term, $P_{j}(t)$, is the same as in equation 2.2. Only neighboring oscillators that both receive stimulus have a nonzero coupling strength. The connection strengths are normalized so that all stimulated oscillators receive the same sum of connections and thus have the same frequency. However, we use a slightly modified version of equation 2.3 to reflect that the input to oscillator $i$ is now normalized by the number of stimulated neighbors coupled with $i$. All of the above can be neurally implemented by dynamic normalization of neural connections (Wang, 1995).

The global inhibitor, $G(t)$, sends an instantaneous inhibitory pulse to the entire network when any oscillator in the network fires. It is defined as

$$
G(t)=\Gamma \delta\left(t-t_{j}^{m}\right), \quad \forall j, m,
$$

where $t_{j}^{m}$ represent the $m$ firing times of the $j$ th oscillator. The constant $\Gamma$ is less than the smallest coupling strength between neighboring oscillators. 
A

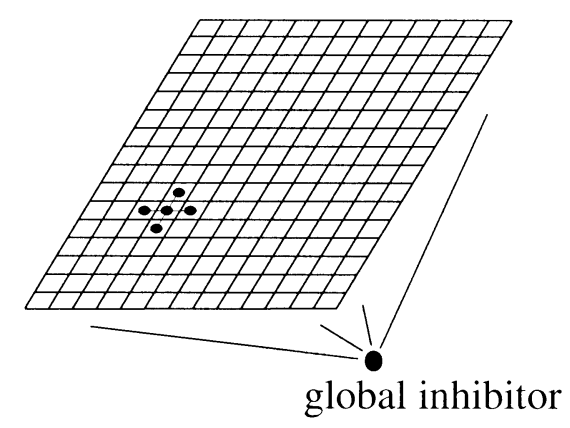

B

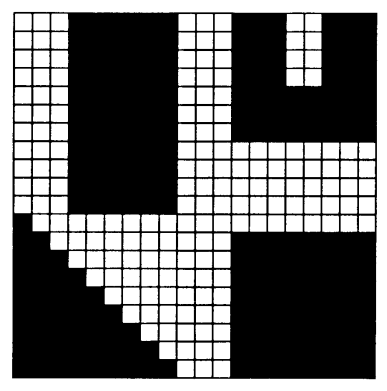

$\mathrm{C}$

rectangle
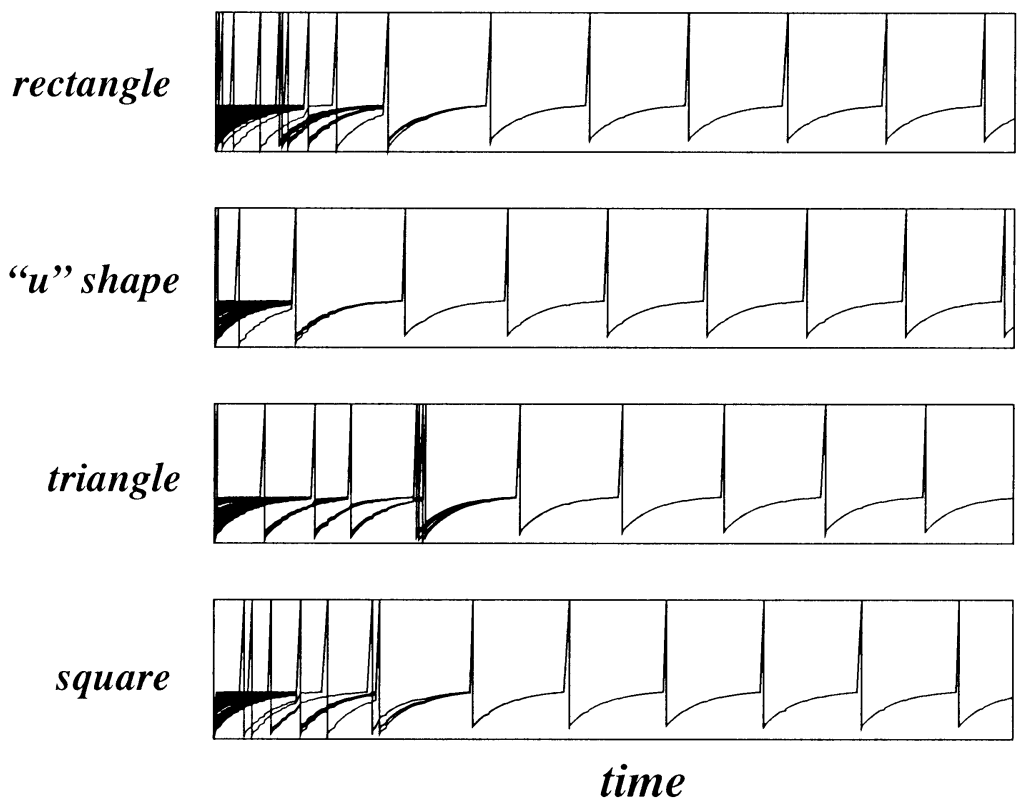

Figure 7: (A) Diagram of the network architecture. Each oscillator has local excitatory connections. The global inhibitor is coupled with every oscillator in the network. (B) Input image. The black squares represent those oscillators that receive stimulus, and the oscillators corresponding to the white squares receive no stimulus. (C) Temporal activities of all units comprising each of the four objects in (B). The parameters are $I_{i}=1.05$ for oscillators receiving stimulus, $\alpha=0.2$, and $\Gamma=0.01$. 
When an oscillator fires, the global inhibitor serves to lower the potential of all oscillators, but because this impulse is not as large as the excitatory signal between neighboring oscillators, it does not destroy the synchronizing effect of the local couplings (see Terman \& Wang, 1995). In this fashion, a connected region of oscillators receiving input synchronizes as the system evolves in time. This region of oscillators has no direct excitatory connections with other spatially separate regions of oscillators. It will, however, interact with other groups through the global inhibitor. This interaction inhibits other blocks of oscillators from firing at the same time.

We now demonstrate the ability of this network to perform oscillatory correlation. In Figure 7B we display an input image with four objects and in Figure $7 \mathrm{C}$ the network response. The four graphs in Figure $7 \mathrm{C}$ display the combined potentials of all the oscillators comprising each of the four objects. The oscillators have random initial conditions varying uniformly from 0 to 1 . Initially many oscillators fire; the effect of the global inhibitor can be seen in the jitter, or lack of smoothness, in the potentials of the oscillators during this time. As the system evolves, clusters of oscillators begin to form, and the curves become smoother because the global inhibitor does not send inhibitory impulses as often. By the third cycle, each group of oscillators comprising a distinct object is almost perfectly synchronous, and the different oscillator groups have distinct phases. Oscillators that do not receive excitation (not shown) experience an exponential decay toward zero and are periodically perturbed by the small inhibitory signals from the global inhibitor.

In this network, there is an unlimited number of oscillator groups that can be segmented. In other words, the segmentation capacity is infinite. Imagine two groups of oscillators that have nearly the same phase. When the first group fires, the potential of the second group of oscillators decreases by $\Gamma$. Thus, the second group needs to traverse the distance $\Gamma$ before it can fire. This implies that there is a finite amount of time between the firings of two consecutive groups. This also implies that as the number of groups increases, the period of the system increases. Simulations support the above statements, and we have segmented more than 100 groups of oscillators.

\section{Image Segmentation}

In the previous section, we segmented four black objects on a white background in a $20 \times 20 \mathrm{image}$. Since our study suggests that there is a logarithmic scaling relation between the time to synchrony and the network size, we expect to be able to use this same network to perform image processing tasks with much larger images quickly.

In order to segment gray-level images, we alter how the connection weights and values of $I_{i}$ are chosen. The alterations are variations of the methods proposed in Wang and Terman (1997). Let the intensity of pixel $i$ be denoted by $p_{i}$. If $\left|p_{i}-p_{j}\right|$ is less than a given threshold, then the two pixels 
are said to satisfy the pixel difference test. Two oscillators have a nonzero coupling strength only if they are neighbors (we now use the eight nearest neighbors of $i$ ) and if their corresponding pixel values satisfy the pixel difference test. The weights of the connection strengths are determined using equation 2.3, except that $Z_{i}$ now represents the number of neighboring pixels of $i$ that pass the pixel difference test. The stimulus $I_{i}$ for each oscillator is chosen in the following manner. We examine a region $Q(i)$ centered on pixel i. $Q(i)$ is a neighborhood about oscillator $i$ that contains more pixels than $N(i)$. If half of the pixels in $Q(i)$ satisfy the pixel difference test, then pixel $i$ is likely within a homogeneous region, and we set the stimulus, $I_{i}$, to a value $I_{L}$, which is greater than 1 . Such an oscillator is called a leader (Wang \& Terman, 1997) and is able to oscillate by itself. If $Q(i)$ contains no pixels that satisfy the pixel difference test, the corresponding oscillator receives no stimulus and does not oscillate. Otherwise oscillator $i$ is given a stimulus $I_{N}$, which is less than but near 1 and is said to be a near-threshold oscillator. A near-threshold oscillator is able to fire only through interactions with other oscillators. In this fashion, only regions of sufficient size and with smoothly varying intensities will contain leaders. These leaders will oscillate and can induce neighboring oscillators that are near threshold to oscillate. Regions with high-intensity variations will not exhibit oscillatory activity and are referred to as the background.

The rules for the connection weights and oscillator stimuli described above have been implemented in an integrate-and-fire oscillator network, and we display the segmentation results for two real images in Figure 8. Figure 8A displays an aerial photograph. In Figure 8B we display the segmentation results of our network. Each group of synchronous oscillators is represented by a single gray-level intensity. Inactive oscillators comprising the background are colored black. There are 29 regions segmented, although it is not easy to discern every different gray level. We also segment a computerized tomography (CT) image of a slice of a human head. The original gray-level image is shown in Figure 8C. The bright areas indicate bone structure. Our segmentation result is shown in Figure 8D and contains 25 segments. The different bone structures are segmented, except for two of the smaller bones that do not contain many pixels. Regions of soft tissue are also segmented.

Our demonstration here is not meant to be a claim that we produce better segmentation results with these images. Rather, our objective is to illustrate the utility of integrate-and-fire oscillator networks for such tasks. The distinctive feature of such networks for image segmentation includes its neurobiological basis and its parallel and distributed nature of computation.

\section{Discussion}

We have investigated the time complexity of synchronization, in particular the scaling relation between the time to synchrony and the system size, in 
A

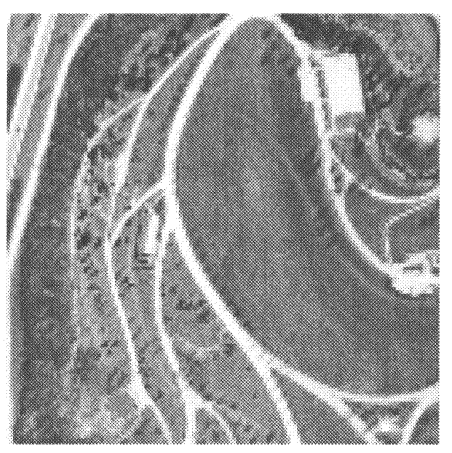

B

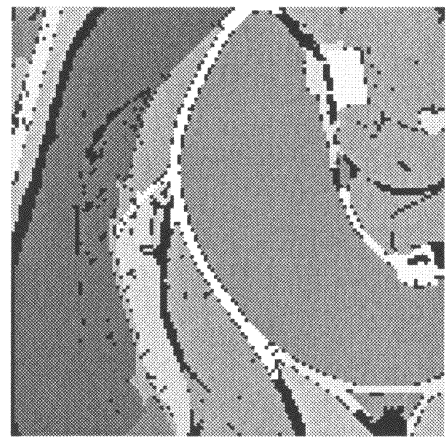

C

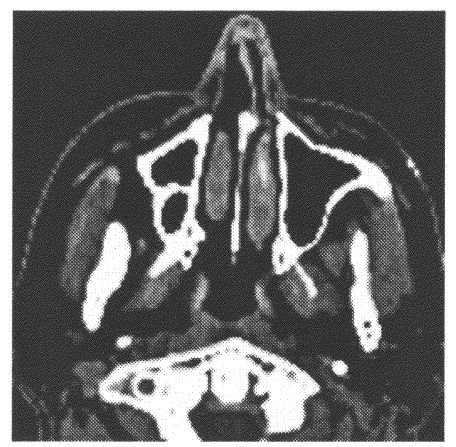

D

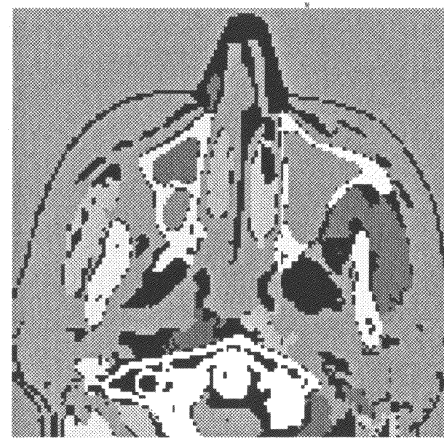

Figure 8: (A) Aerial image with $128 \times 128$ pixels. (B) Segmentation results for (A). The network produced 29 different synchronized groups. Each synchronized group is represented by a single gray level. Black pixels represent oscillators that do not oscillate. The threshold for the pixel difference test is $19, Q_{i}$ is a region of size $7 \times 7$, with $I_{L}=1.025, I_{N}=0.99, \alpha=0.2$, and $\Gamma=0.01$. (C) $128 \times 128 \mathrm{CT}$ image of a slice of a human head. (D) Segmentation results for (C). The network produced 25 different groups of synchronized oscillators. The threshold for the pixel difference test is $15, Q_{i}$ is a region of size $9 \times 9$, and the other parameters are as listed above.

locally coupled networks of integrate-and-fire oscillators. Our data strongly suggest that $1 \mathrm{D}$ and 2D systems of identical oscillators synchronize at times proportional to the logarithm of the system size. We have also given an approximation relating the rate of synchronization to the system parameters. Remarkable rates of synchronization can be achieved. For example, one can choose parameters so that a chain of $10^{6}$ oscillators can synchronize in approximately six cycles. This is opposite to the conclusion reached by Hopfield and Herz (1995), who discount global synchrony in such networks as too slow to be useful in biological computations, and instead use 
networks capable of fast local synchrony to perform computations. In local synchrony, small clusters of oscillators fire at the same time, and the entire network may consist of many such clusters.

We also used integrate-and-fire oscillators to create an oscillator network that performs oscillatory correlation. We found that using the LEGION architecture (Terman \& Wang, 1995), we were able to create a global inhibitor that serves the purpose of desynchronizing different groups of oscillators while maintaining synchrony within each group of oscillators. Our segmentation network is different in several ways from that proposed for image segmentation by Hopfield and Herz (1995). One difference is in terms of encoding. In our network, relations among pixels are encoded into coupling strengths between neighboring oscillators. In contrast, in the Hopfield and Herz network, pixel relations are encoded into initial phases of oscillators. Another difference is that the Hopfield and Herz network does not actively desynchronize oscillator groups. Two regions with the same gray level fire at the same time in their network. Our network actively desynchronizes groups of oscillators so that no two groups can fire at the same time. The process of desynchronization eliminates one possible source of mistakes during segregation: accidental synchrony, which refers to synchrony between oscillator blocks that have no intrinsic relations (Hummel \& Biederman, 1992).

A major difference in image segmentation between our network and that studied by Terman and Wang (1995) is the capacity of segmentation, or the number of different objects that can be desynchronized. Their network has a distinct limit on the number of groups that can be desynchronized. This limit is directly related to the ratio between the amount of time a relaxation oscillator spends in the silent (low-activity) phase of the limit cycle in comparison to that spent in the active (high-activity) phase of the limit cycle (Wang \& Terman, 1997). However, with integrate-and-fire oscillators this ratio is essentially infinite, because the firing of a spike takes place instantaneously and such an oscillator does not have a finite active phase as does a relaxation oscillator. In our integrate-and-fire network, when a group of oscillators fires, the amplitudes of all other groups are instantly decreased by some amount. In essence, this increases the period as the number of groups increases. Since there is no consequence of lengthening the period, there is no limitation on the number of groups that can be desynchronized. Thus the concept of segmentation capacity is not relevant in our network, or one may regard our network as having an infinite capacity of segmentation.

One important topic in networks of neural oscillators is the inclusion of time delays in the connections between oscillators. Like numerous other studies on integrate-and-fire oscillators, our model does not include conduction delays. However, several studies have examined time delays in networks of integrate-and-fire oscillators. Ernst, Pawelzik, and Geisel (1995) showed that a time delay in the excitatory connections between two oscil- 
lators leads to a difference in their firing times: no synchrony. We have confirmed this result in our simulations. Our preliminary results in locally coupled integrate-and-fire oscillators with time delays further indicate that although the system may not reach perfect synchrony, the firing times of neighboring oscillators are highly correlated. In a related study on relaxation oscillator networks with similar coupling structure, Campbell and Wang (1998) showed that loose synchrony, instead of perfect synchrony, occurs whereby neighboring oscillators converge to a phase difference within the conduction delay. Interestingly, if the coupling is changed from excitatory to inhibitory, two coupled integrate-and-fire oscillators can be perfectly synchronous (van Vreeswijk, Abbott, \& Ermentrout, 1994; Ernst et al., 1995). Synchronization in inbibitory networks of integrate-and-fire oscillators with all-to-all couplings and conduction delays is discussed in Ernst et al. (1995) and Gerstner, van Hemmen, and Cowan, (1996).

Understanding the scaling relation between the time to synchrony and the network size is a complex and intriguing issue. Diffusively coupled phase oscillators synchronize at times proportional to the length of the system squared (Niebur, Schuster, Kammen, \& Koch, 1991) and relaxation oscillators with a Heaviside coupling are conjectured to synchronize at times proportional to the length of the system (Somers \& Kopell, 1993). We believe that it is important to understand how the type of oscillator and the type of interaction between oscillators are related to various scaling relations.

\section{Appendix}

As used in the text, we introduce in 1D systems a domain wall between any two adjacent synchronized oscillator blocks. In this appendix, we prove the following theorem.

Theorem. In a one-dimensional network of integrate-and-fire oscillators, as defined in equations 2.1 through 2.4, the number of domain walls or, equivalently, the number of synchronized oscillator blocks does not increase.

Proof. Given the definitions of $f$ and $g$, we have the following two facts:

1. Given $f^{\prime}(\phi)>0$ and $f^{\prime \prime}(\phi)<0$, the potential difference between two oscillators shrinks monotonically when their phases advance, assuming that no pulse is generated or received by either oscillator.

2. Given $g^{\prime}(x)>0$ and $g^{\prime \prime}(x)>0$, it follows that $g(x+\alpha / 2)-g(x)>$ $g(y+\alpha / 2)-g(y)$ if $x>y$, and $x+\alpha / 2 \leq 1$.

Consider a synchronized block of oscillators. The theorem is proved if we can prove that either no new domain wall is created within this block, or when a new domain wall is created, another existing domain wall disappears. The latter case corresponds to a shift of a domain wall. In order to 
create a domain wall within the block, the block size must be greater than 1 . Let us first consider the case of the block size greater than 2. In this case, there is at least one interior oscillator. Let the block fire at $t=0$. Immediately afterward at $t=0^{+}$, all the interior oscillators receive two pulses due to local excitation, whereas the two exterior (boundary) oscillators receive one pulse. Thus, we have

$$
\begin{aligned}
& \alpha / 2 \leq x_{i} \leq \alpha \quad \text { if } i \text { is an interior oscillator } \\
& 0 \leq x_{i} \leq \alpha / 2 \text { if } i \text { is an exterior oscillator }
\end{aligned}
$$

When the oscillators in the block fire again, there are two possible situations:

1. The first oscillator (leading) to fire again is an interior one, at $t=t_{1}$. When the leading oscillator fires, all interior oscillators are in the jumping region due to equation A.1a and fact 1 . Thus no domain wall is created in the interior of the block. Let us consider the possibility of creating a domain wall between an exterior and an interior oscillator. Without loss of generality, consider the right exterior oscillator, denoted as $B$. For $B$ to break away from the block, it must not receive a pulse from its right neighbor, denoted as $C$, in the time period $t \in\left(0, t_{1}\right)$, for otherwise $B$ is in the jumping region at $t=t_{1}$ because of equation A.1b. For this case to occur, $1-\alpha / 2<x_{C}\left(t_{1}\right)<1$ because $x_{C}\left(0^{+}\right)>\alpha / 2$ due to the firing of $B$ at $t=0$. At $t=t_{1}^{+}, B$ receives a pulse from the block and $1-\alpha / 2<x_{B}\left(t_{1}^{+}\right)<1$ due to equation $\mathrm{A} .1 \mathrm{~b}$ and fact 1 . Thus, the firing of either one will synchronize $B$ and $C$, and the domain wall between $B$ and $C$ shifts one site to the left.

The only other case to be considered is that $B$ is at the end of the 1D chain and does not have a right neighbor. In this case, due to weight normalization defined in equation 2.3 in the text, at $t=0^{+}, B$ satisfies $0 \leq x_{B} \leq \alpha$. Again due to equation 2.3, $B$ cannot break away from the block.

2. The leading oscillator to fire again is an exterior one. Without loss of generality, let $B$ be the leading oscillator. If $B$ is at the right end of the chain, $B$ satisfies $0 \leq x_{B} \leq \alpha$ at $t=0^{+}$as discussed above, and when it fires again all the interior oscillators are in the jumping region. The same analysis given in the first situation implies the theorem. Now consider the case that $B$ has a right neighbor. Let $B$ fire at $t=t_{1}$. Because of equation A.1, $B$ must receive a pulse from its right neighbor, $C$, in order to become the leading oscillator. If $B$ receives just one pulse from $C$ at or before $t=t_{1}$, then when $B$ fires, all the interior oscillators of the block are in the jumping region. This is because, in order for $B$ to break away, the most favorable time for $B$ to receive a pulse from $C$ is when $t=t_{1}$ (see fact 2). Even in this case, the interior oscillators are 
in the jumping region because of equation A.1. The same argument given in the first situation also ensures that the other exterior oscillator either remains in the block at $t=t_{1}$ or joins the block to its left (a shift of the domain wall). Thus, the proof is completed if we can prove that $B$ cannot receive more than one pulse from $C$ during $t \in\left(0, t_{1}\right)$. If $t_{1} \geq g(1-\alpha / 2)-g(\alpha / 2)$, then all the interior oscillators are in the jumping region due to equation A.1a, and the theorem is established by the above argument. Thus, the proof is completed if the following proposition is true.

Proposition. In the period $T=(0, g(1-\alpha / 2)-g(\alpha / 2)), C$ cannot fire more than once.

Proof. Using proof by contradiction. Assume that $C$ can fire at least twice during $T$. Without loss of generality, we examine the possibility of $C$ firing twice. The best scenario for $C$ to produce two pulses is when $C$ generates a pulse shortly after the block fires, at $t=0^{++}$. Since $C$ is not in the same block, after $C$ fires and resets at $t=0^{++}, x_{C}\left(0^{++}\right) \leq \alpha / 2$. If $C$ receives just one pulse from its right neighbor during $T, C$ cannot produce two pulses by a similar argument. Thus, in order for $C$ to fire twice, it must receive two pulses from its right neighbor, denoted by $D$ during $t \in\left(0^{+}, g(1-\alpha / 2)-g(\alpha / 2)\right)$. Note that $D$ cannot receive a pulse from $C$ during this time period. There are two possible cases to consider for $D$ :

1. $D$ is not in the same block as $C$. The same argument leads to the requirement that $D^{\prime}$ s right neighbor, denoted by $E$, must receive two pulses from $E^{\prime}$ s right neighbor.

2. $D$ is in the same block as $C$. Let us call this block the $D$ block. If $E$ is not in the $D$ block, then at $t=0^{++}, x_{D}\left(0^{++}\right) \leq \alpha / 2$. The same argument again leads to the same requirement that $E$ must receive two pulses from its right neighbor. If $E$ is in the $D$ block, then $D$ becomes an interior oscillator, bounded by equation A.1a at $t=0^{++}$. Before $t=g(1-\alpha / 2)-g(\alpha / 2)$, no interior oscillator of the $D$ block can be a leading oscillator of the block because of fact 2 . The only possible way for $D$ to jump before $t=g(1-\alpha / 2)-g(\alpha / 2)$ is to have the right exterior oscillator, $B^{\prime}$, of the $D$ block to be the leading oscillator of the block. But at $t=0^{++}, B^{\prime}$ is bounded by $x_{B^{\prime}} \leq \alpha / 2$, and it cannot jump before $t=g(1-\alpha / 2)-g(\alpha / 2)$ without receiving two pulses from its right neighbor. Thus we are back to the same requirement.

The analysis indicates a pattern of cyclic requirement. It is straightforward to show that the oscillator at the right end of the entire chain cannot produce two pulses during $T$. Thus, the cyclic requirement cannot be satisfied, and the proposition is proved. 
The proposition completes the proof of the theorem for the case of the block size greater than 2 . If the block size equals 2 , we note that both oscillators in the block satisfy equation A.1b at $t=0^{+}$. It is easy to show that the theorem holds for this case as well. Thus, we complete the proof.

\section{Acknowledgments}

We are grateful to E. Cesmeli, who provided much assistance in preparing the manuscript, and three anonymous referees whose constructive suggestions have improved the article. This work was supported by an ONR grant (N00014-93-1-0335), an NSF grant (IRI-9423312), and an ONR YIP Award (N0014-96-1-0676) to D. L. W.

\section{References}

Campbell, S. R. (1997). Synchrony and desynchrony in neural oscillators. Unpublished doctoral dissertation. Ohio State University, Columbus.

Campbell, S. R., \& Wang, D. L. (1998). Relaxation oscillators with time delay coupling. Physica D, 111, 151-178.

Corral, A., Perez, C. J., Diaz-Guilera, A., \& Arenas, A. (1995). Self-organized criticality and synchronization in a lattice model of integrate-and-fire neurons. Phys. Rev Let., 74, 118-121.

Ernst, U., Pawelzik, K., \& Geisel T. (1995). Synchronization induced by temporal delays in pulse-coupled oscillators. Phys. Rev. Lett., 74, 1570-1573.

Gerstner, W., van Hemmen, J. L., \& Cowan, J. D. (1996). What matters in neuronal locking? Neural Comp., 8, 1653-1676,

Hopfield, J. J., \& Herz, A. V. M. (1995). Rapid local synchronization of action potentials: Toward computation with coupled integrate-and-fire oscillator neurons. Proc. Natl. Acad. Sci. USA, 92, 6655-6662.

Hummel, J., \& Biederman, I. (1992). Dynamic binding in a neural network for shape recognition. Psychol. Rev., 99, 480-517.

Livingstone, M. (1996). Oscillatory firing and interneuronal correlations in squirrel monkey striate cortex. J. Neurophysiol., 75, 2467-2485.

Milner, P. M. (1974). A model for visual shape recognition. Psych. Rev., 81, 521535.

Mirollo, R. E., \& Strogatz, S. H. (1990). Synchronization of pulse-coupled biological oscillators. SIAM J. Appl. Math., 50, 1645-1662.

Niebur, E., Schuster, H. G., Kammen, D. M., \& Koch, C. (1991). Oscillator-phase coupling for different two-dimensional network connectivities. Phys. Rev. A, 10, 6895-6904.

Peskin, C. S. (1975). Mathematical aspects of heart physiology. New York: New York University Courant Institute of Mathematical Sciences.

Singer, W., \& Gray, C. M. (1995). Visual feature integration and the temporal correlation hypothesis. Ann. Rev. of Neurosci., 18, 555-586.

Somers, D., \& Kopell, N. (1993). Rapid synchronization through fast threshold modulation. Biol. Cybern., 68, 393-407. 
Sporns, O., Tononi, G., \& Edelman, G. (1991). Modeling perceptual grouping and figure-ground segregation by means of active re-entrant connections. Proc. Natl. Acad. Sci. USA, 88, 129-133.

Terman, D., \& Wang, D. (1995). Global competition and local cooperation in a network of neural oscillators. Physica D, 81, 148-176.

Traub, R., Whittington, M., Stanford, M., \& Jefferys, J. (1996). A mechanism for generation of long-range synchronous fast oscillations in the cortex. Nature, $383,621-624$.

van Vreeswijk, C., Abbott, L. F., \& Ermentrout, B. (1994). When inhibition not excitation synchronizes neural firing. J. Comp. Neurosci., 1, 313-321.

von der Malsburg, C. (1981). The correlation theory of brain functions. (Internal Rep. No. 81-2.) Max-Planck-Institute for Biophysical Chemistry, Göttingen, FRG.

Wang, D. L. (1993). Modeling global synchrony in the visual cortex by locally coupled neural oscillators. In Proc. 15th Ann. Conf. Cognit. Sci. Soc. (pp. 10581063).

Wang, D. L. (1995). Emergent synchrony in locally coupled neural oscillators. IEEE Trans. Neural Net., 6, 941-948.

Wang, D. L., \& Terman, D. (1995). Locally excitatory globally inhibitory oscillator networks. IEEE Trans. Neural Net., 6, 283-286.

Wang, D. L., \& Terman, D. (1997). Image segmentation based on oscillatory correlation. Neural Comp., 9, 805-836. (For errata see Neural Comp., 9, 16231626, 1997.)

Zeki, S. (1993). A vision of the brain. Oxford: Blackwell.

Received February 4, 1998; accepted December 10, 1998. 